\title{
Characterization and Antibiotic Susceptibility of Bacteria Isolated from Clinical Specimens in Rajshahi, Bangladesh
}

\author{
Shekha Nita Mondal', Md. Ariful Islam ${ }^{1,2}$, Tamanna Zerin'1 \\ ${ }^{1}$ Department of Microbiology, Stamford University Bangladesh, 51, Siddeswari Road, Dhaka 1217, Bangladesh. \\ ${ }^{2}$ Rajshahi Metropolitan Hospital Ltd. and Diagnostic Center, C \& B Mor, Laxmipur, Kajihata, Rajpara, Rajshahi \\ 6000, Bangladesh \\ Corresponding Author: Tamanna Zerin
}

DOI: https://doi.org/10.52403/ijhsr.20220203

\begin{abstract}
Infectious diseases caused by antibiotic-resistant infectious bacteria are a major threat to human survival and development. This is a retrospective study for a one-year period from January 2020 to December 2020 with a total of 1,112 urine, 318 blood, 167 stool, and 72 pus samples conducted in the Microbiology Laboratory of Rajshahi Metropolitan Hospital Ltd. and Diagnostic Center, Rajshahi, Bangladesh. Bacterial isolation, characterization, and antibiotic susceptibility were performed following the standard microbiological methods. Out of 1112 urine, 318 blood, 167 stool, and 72 pus specimens, bacteria were detected in 345 urine, 86 blood, 44 stool, and 50 pus specimens, respectively. Specimens from females $(57.10 \%)$ were found more positive than those from males $(42.90 \%)$ only in the case of urine samples. Among all the specimens, the highest number of infections was recorded in the age group 31-40, except in stool, where the highest positive cases were evidenced in the 0-10 age group. The greatest number of specimens was received, mostly in the summer and rainy seasons. However, E. coli bacteria were the most common among all the specimens excluding pus, where Staphylococcus aureus was the highest in number. Gram-positive and gramnegative bacteria showed higher sensitivity towards Cefepime (against pathogens of blood and stool samples) along with Imipenem, and Meropenem (pathogens of both urine and pus samples). A terrifying scenario was the high degree of resistance of those isolated bacteria towards the $3^{\text {rd }}$ and $4^{\text {th }}$ generation cephalosporin antibiotics. This study might be helpful for physicians to rationalize empiric treatment strategies and provide awareness among government authorities and the population.
\end{abstract}

Keywords: Bangladesh, Clinical specimens, Resistance, Retrospective, Sensitivity.

\section{INTRODUCTION}

Infectious diseases (IDs) pose a significant burden on human health and survival. Infectious diseases are estimated to cause at least $25 \%$ of the approximately 60 million deaths that occur worldwide each year (1). However, increasing antibioticresistant bacteria due to conscious and unconscious use of antibiotics is an emergent public health concern. Among bacterial infections, urinary tract infections (UTIs) (usually caused by E. coli,
Klebsiella, Pseudomonas, and Enterococcus spp.), bloodstream infections (BSIs) (can be caused by Salmonella spp., Staphylococcus aureus, S. epidermidis, Pseudomonas, E. coli, etc.), wound infections (may be associated with Staphylococcus aureus, S. epidermidis, Methicillin-resistant Staphylococcus aureus (MRSA), E. coli, Klebsiella pneumoniae, Pseudomonas aeruginosa, Proteus vulgaris, Proteus mirabilis, Enterococcus spp., Enterobacter spp. and Acinetobacter spp.), diarrheal 
diseases and enteric infections (major causative agents are Salmonella spp. and Shigella spp.) are common in the human population $(2,3)$. Many different types of laboratory tests use a sample of blood, urine, sputum, pus, or other fluid/tissue from the body to identify microorganisms. Sometimes several different tests are done to identify every microorganism because tests that work well for one microorganism often do not work well for another (4). Among several tests, culture is one of the most important tools for their diagnosis (2). Although physicians know in general which antimicrobial drugs are effective against different microorganisms, the problem of the emergence of drug-resistant microbes very well characterizes many bacterial infectious agents such as Escherichia coli, Pneumococcal, Neisseria gonorrhoeae, Staphylococcus aureus, and so on. Many well-known antibiotics no longer clear bacterial infections due to microbial resistance $(1,4)$. Resistance to antibiotics is accelerated by the misuse, overuse, and overly prescribed antibiotics which leads to higher medical costs, prolonged hospital stay, and increased mortality (5). Thus, susceptibility testing (culture) is done to determine how effective various antimicrobial drugs are against the specific microorganism infecting the person. This testing helps physicians in determining which drug to use for a particular person's infection (4). Many medical advances are dependent on the ability to fight infections using antibiotics, including joint replacements, organ transplants, cancer therapy, and treatment of chronic diseases like diabetes, asthma, and rheumatoid arthritis (6). Therefore, our study was aimed to detect the season-, gender- and age-wise distribution of infected patients, identify the pathogenic microbes from infected patients, and analyze the sensitivity of isolated bacteria from clinical specimens towards commonly prescribed antibiotics in that region.

\section{MATERIALS AND METHODS Study Area, Period, and Population}

This study was carried out in the Microbiology Laboratory of Rajshahi Metropolitan Hospital Ltd. and Diagnostic Center which is located in C \& B Mor, Laxmipur, Rajshahi, Bangladesh over a period from January 2020 to December 2020. A total number of 1,112 samples of urine from clinically suspected UTI patients, 318 samples of blood from clinically suspected patients with bacteremia, 167 samples of stool from clinically suspected patients with having infections of the digestive tract, and 72 samples of pus from clinically suspected patients with wound infection were evaluated for our study. The standard microbiological methods were followed during the study period.

\section{Sample collection}

In brief, midstream urine samples were collected in a wide-necked sterile screw-cap container labelled with the date, time, patient's Id, and name of the patients. In collecting blood samples, 1-2 $\mathrm{ml}$ and 5$10 \mathrm{ml}$ of venous blood were aspirated and directly incorporated into blood culture bottles aseptically in pediatric and adult cases, respectively. To collect the stool sample, a clean screwtop container with the patient's name, date, time, and patient's Id was used.

For dry wounds, the specimen was collected with two cotton-tipped swabs moistened with sterile non-bacteriostatic saline while in the case of moist pus, the sterile swab was used to collect it from a superficial wound site. For a deeper wound, the fluid was aspirated into a syringe which was aseptically transferred into a sterile specimen container.

Samples were processed and cultured within $2 \mathrm{~h}$ of sample collection and in certain cases, they were stored at $4^{\circ} \mathrm{C}$ for less than $24 \mathrm{~h}$.

\section{Process of culturing samples}

A calibrated loop was used to transfer urine for culturing uropathogens. To 
culture, $0.1 \mathrm{ml}$ urine sample was spread on Nutrient Agar (NA) (Oxoid) and MacConkey Agar (MAC) (Oxoid) media following standard bacteriological technique. After inoculation, all the plates were incubated aerobically at $37^{\circ} \mathrm{C}$ for 18 $24 \mathrm{~h}$ and following incubation, those were examined in terms of the number of colonies with colony morphology. The samples were considered positive when the colony number was $\geq 105$ colony forming unit $(\mathrm{CFU}) / \mathrm{ml}$.

To culture, blood culture bottles were incubated at $37^{\circ} \mathrm{C}$ aerobically for visible growth to come. Following visible growth, 2-3 drops of the blood culture were inoculated on Blood Agar (BA) (Oxoid) and MAC agar (Oxoid) media. Blood culture bottles that do not show any significant growth till the 7 th day of incubation were reported as culture negative.

Stool specimens were inoculated onto MAC agar, Salmonella-Shigella agar (SS) (Oxoid) which were incubated overnight at $37^{\circ} \mathrm{C}$. If no growth, the culture is considered negative but if there is growth, the culture is positive.

Pus samples were inoculated onto $\mathrm{BA}$ and MAC agar media at $37^{\circ} \mathrm{C}$ for $24 \mathrm{~h}$ and growth was observed. If there is no growth, the plates were incubated for a further $24 \mathrm{~h}$.

\section{Identification of microbial isolates}

All the colonies were sub-cultured onto fresh media to get pure culture. Microbial identification was performed as per the standard protocol followed in the microbiology laboratory. Microbial and colony morphology along with the conventional biochemical test results were recorded and bacterial identification was performed by standard protocol followed in the microbiology laboratory (7).

\section{Antibiogram of bacterial isolates}

Modified Kirby-Bauer disc diffusion method was followed to identify the sensitivity pattern of the isolates. In brief, isolated colonies were placed over MuellerHinton Agar (MHA) media and then, paper discs containing antibiotics were placed on top of it. Following incubation at $37^{\circ} \mathrm{C}$ for $24 \mathrm{~h}$, the zone of inhibitions (ZOIs) was measured to detect the sensitivity or resistance of bacteria by comparing with the Clinical and Laboratory Standard Institute (CLSI) guideline (8). However, commercially available antibiotics that were available in the laboratory in time were considered for antibiogram study.

\section{RESULTS}

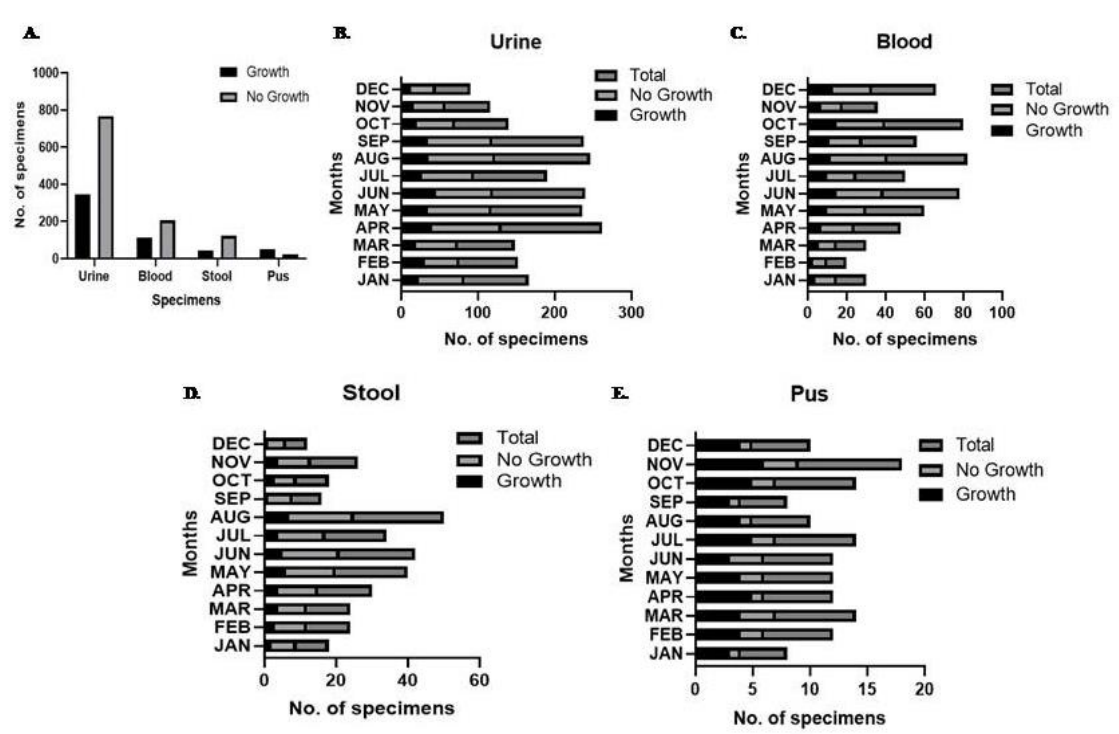

Figure 1: The bar graph presented the total number of growth and no growth of urine, blood, stool, and pus specimens (A), and month-wise distribution of total, growth, and no growth in urine (B), blood (C), stool (D), and pus (E) specimens. 
It was a one-year study that was carried out from January 2020 to December 2020 with a total number of 1112 urine, 318 blood, 167 stool, and 72 pus samples. According to figure $1 \mathrm{~A}$, the number of collection of urine specimens was the highest while pus specimens were the lowest among the 4 specimens (urine, blood, stool, and pus). Though the number of pus samples was the lowest, the positive cases of pus samples were the highest which accounts for around $69.44 \%$. The monthwise distribution of growth positive and negative samples from figure $1 \mathrm{~B}$ described that the bacterial growth in urine samples was top in June but relatively at the bottom in December. On the other hand, pathogenic growth (positive cases) from blood samples was upright in June and October while it was the lowest in February (Figure 1C). The bacterial growth (positive cases) in stool samples elevated in August and demoted in September and December (Figure 1D), while bacterial growth (positive cases) in pus samples was the highest in November and the lowest in January, June, and September (Figure 1E).
On the basis of figure $2 \mathrm{~A}$, the number of positive cases of females (197) was higher than males (148) in the case of urine samples where positive cases of males (65 in case of blood, 26 in stool, and 34 in pus samples) were upper than females (48 in blood, 18 in stool and 16 in pus) among 3 other specimens. The bar chart illustrated the age-wise distribution of both positive female and male cases which was divided into 6 different categories as 0-10, 11-20, 21-30, 31-40, 41-50, and 51-above ages throughout the study period (Figure 2B, 2C, $2 \mathrm{D}$, and $2 \mathrm{E}$ ). Overall, it was found that the number of infections was the highest at the age between 31 to 40 years in the case of urine, blood, and pus samples (Figure 2B, $2 \mathrm{C}$, and $2 \mathrm{E}$ ). Nevertheless, the number of infections in stool samples was very high in the early ages especially in $0-10$ years compared to other age groups (Figure 2D). In the case of pus specimens, the infected number of patients was the lowest at the age group 0-10 years (Figure 2E). All the infections were occurred due to single culture.

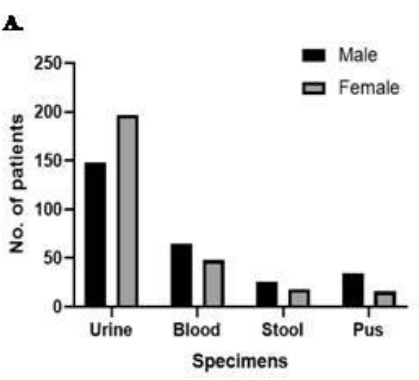

B.
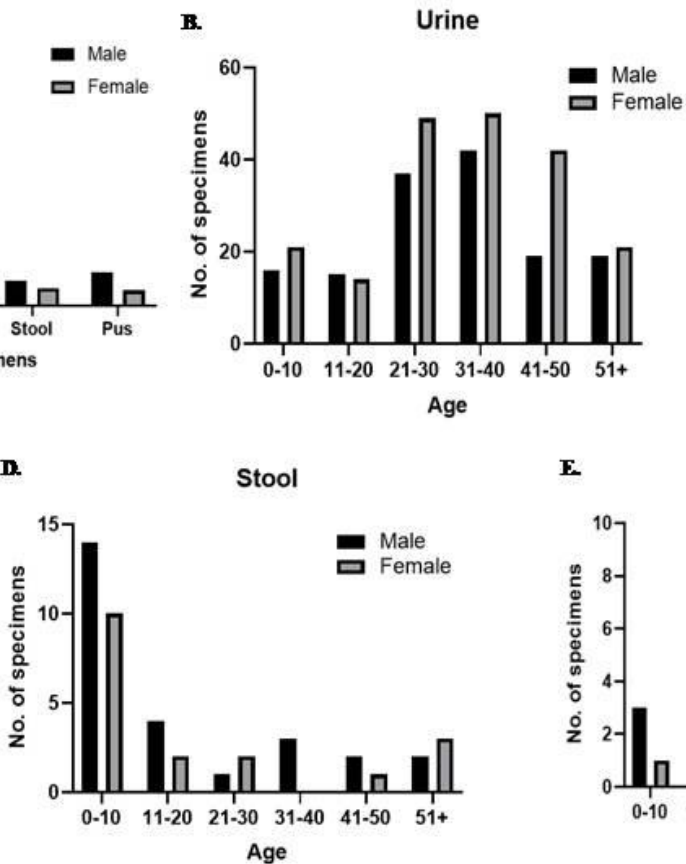

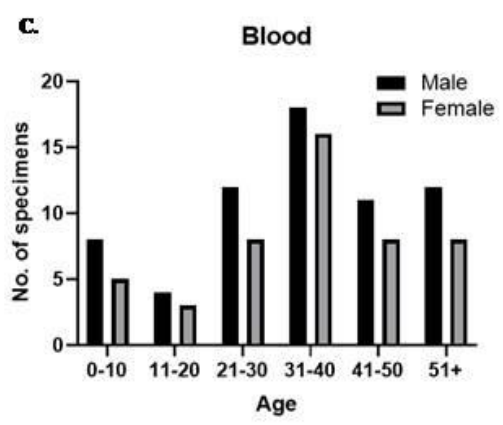

E.

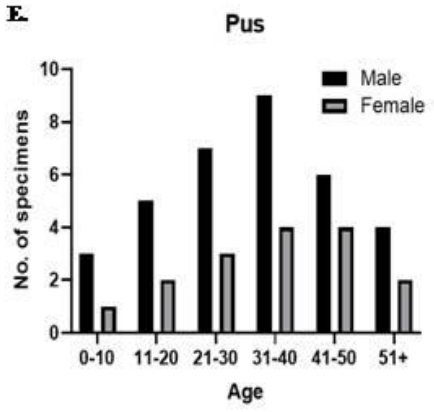

Figure 2: Gender-wise distribution of overall positive cases in all the four types of specimens (A). Age- and Gender-wise distribution of positive specimens in urine (B), blood (C), stool (D), and pus (E) samples. 
The most frequently found bacteria were $E$. coli in urine, blood, and stool specimens regardless of any gender (Figure 3A, 3B, and 3C). Contrastingly, Staphylococcus aureus was recorded as very high in number in pus samples (Figure 3D). Besides those predominating bacteria found in the specimens, Klebsiella spp., Pseudomonas spp., Citrobacter spp., and Enterococcus spp. were found in urine
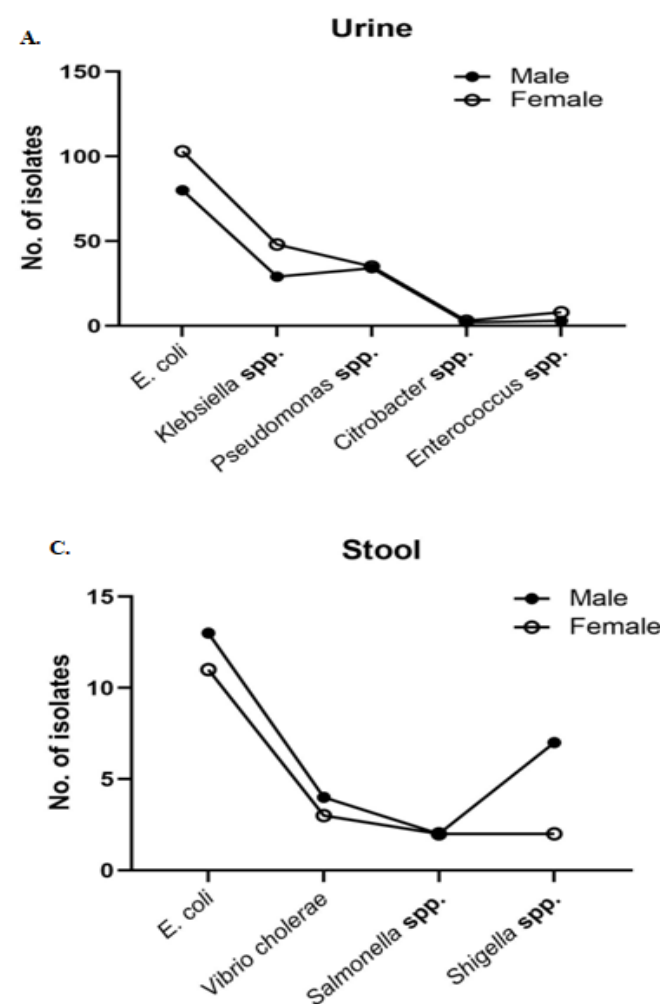

samples; Klebsiella pneumoniae, Salmonella spp., Streptococcus viridans, Staphylococcus epidermidis, Staphylococcus aureus, and Candida albicans in blood samples; Vibrio cholerae, Salmonella spp. and Shigella spp. in stool samples; and E. coli, Pseudomonas spp., Klebsiella spp., Acinetobacter spp. in pus specimens (Figure 3).
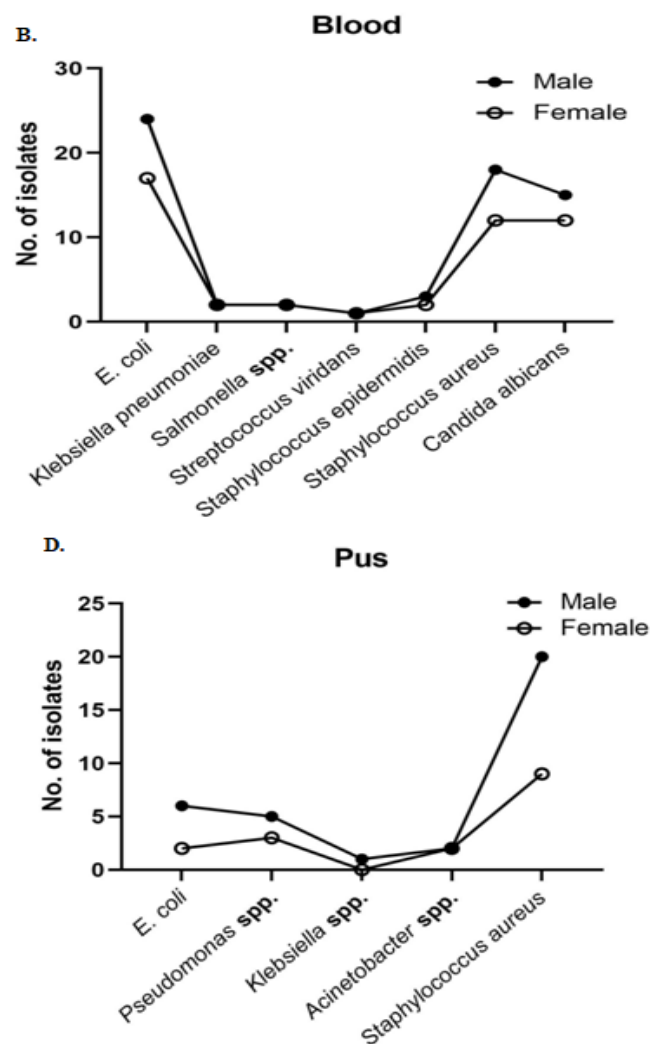

Figure 3: Occurrence of bacterial isolates in urine (A), blood (B), stool (C), and pus (D) specimens of infected male and female patients.

Our antibiogram data showed higher sensitivity towards Imipenem, and Meropenem against pathogens of urine and pus samples; Meropenem, Imipenem, and Cefepime against pathogens of blood samples; and Meropenem, and Cefepime against pathogens of stool samples. No pathogens were found completely sensitive or resistant to all the antibiotics. Most of the bacteria manifested a degree of sensitivity or resistance towards all the antibiotics. Moreover, all gram-positive and gramnegative bacteria showed a high level of sensitivity for Meropenem, and some of them were also noted as sensitive against both Imipenem and Cefepime. However, the lowest sensitivity was reported towards Nalidixic acid, Cotrimoxazole, and Gentamicin against most of the uropathogens. Most of the isolates in stool samples showed the highest resistance against Ampicillin. In the case of bloodborne pathogens, the lowest sensitivity was observed against Cotrimoxazole in E. coli (20.70\%), against Cefixime in Staphylococcus aureus (31\%), against Ceftazidime in Staphylococcus epidermidis (25\%), against Ciprofloxacin in Klebsiella pneumoniae $(31.80 \%)$, against Cefuroxime sodium in Salmonella spp. and Streptococcus viridans against Cloxacillin $(0 \%)$. In the case of pus isolates, Klebsiella 
spp. against both Erythromycin and Cotrimoxazole (0\%); Pseudomonas spp. against Penicillin (20\%); Staphylococcus aureus against Amikacin (0\%) and Gentamicin (20\%); Acinetobacter against
Cefotaxime (0\%), Gentamicin $(10 \%)$ and Cotrimoxazole (10\%); and E. coli against Amikacin $(0 \%)$ showed the lowest sensitivity by the antibiotic sensitivity pattern analysis (Table 1, 2, 3, and 4).

Table 1: Antibiotic sensitivity of bacterial isolates collected from urine specimens.

\begin{tabular}{|c|c|c|c|c|c|c|c|c|c|c|c|c|}
\hline $\begin{array}{l}\text { Name of } \\
\text { organisms }\end{array}$ & 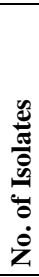 & 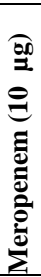 & 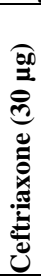 & 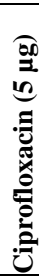 & 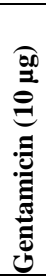 & 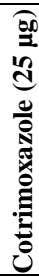 & 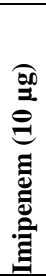 & 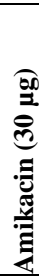 & 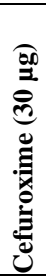 & 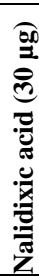 & 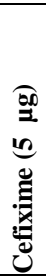 & 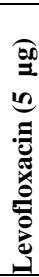 \\
\hline E. coli & $\stackrel{\infty}{\infty}$ & $\begin{array}{l}\stackrel{0}{0} \\
\stackrel{n}{\infty} \\
\dot{\infty}^{\prime}\end{array}$ & $\frac{\circ}{6}$ & $\stackrel{8}{0}^{\circ}$ & 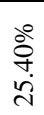 & $\begin{array}{l}\stackrel{2}{\circ} \\
\stackrel{2}{0} \\
\stackrel{2}{0}\end{array}$ & $\begin{array}{l}\stackrel{0}{0} \\
\stackrel{n}{\infty} \\
\stackrel{\infty}{ }\end{array}$ & $\begin{array}{l}\delta^{\circ} \\
\stackrel{\circ}{n} \\
\text { ஸे }\end{array}$ & $\begin{array}{l}\bigotimes^{\circ} \\
\infty \\
i n \\
i n\end{array}$ & $\frac{80}{2}$ & $\frac{\stackrel{\circ}{\gamma}}{\forall}$ & $\begin{array}{l}0 \\
0 \\
\infty \\
0 \\
0 \\
i\end{array}$ \\
\hline $\begin{array}{l}\text { Klebsiella } \\
\text { spp. }\end{array}$ & $\therefore$ & 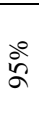 & $\begin{array}{l}\stackrel{0}{0} \\
\text { के } \\
\dot{0}\end{array}$ & $\stackrel{\circ}{\sim}$ & $\begin{array}{l}\stackrel{\circ}{人} \\
\stackrel{2}{2}\end{array}$ & $\stackrel{\stackrel{\circ}{\sim}}{\stackrel{2}{2}}$ & $\begin{array}{l}\delta_{0}^{\circ} \\
\text { ᄋ̊ } \\
\text { ̊̊ }\end{array}$ & $\begin{array}{l}\stackrel{0}{0} \\
\stackrel{0}{0} \\
\dot{f}\end{array}$ & 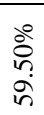 & $\begin{array}{l}\stackrel{0}{0} \\
\stackrel{n}{7} \\
\text { กิ }\end{array}$ & $\begin{array}{l}\stackrel{0}{0} \\
\text { ஜn } \\
\stackrel{n}{n}\end{array}$ & $\begin{array}{l}80 \\
\stackrel{0}{n} \\
\text { ñ }\end{array}$ \\
\hline $\begin{array}{l}\text { Pseudomonas } \\
\text { spp. }\end{array}$ & g & 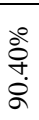 & $\begin{array}{l}\stackrel{8}{0} \\
\stackrel{0}{\circ} \\
\stackrel{0}{0}\end{array}$ & $\stackrel{\stackrel{\Im}{N}}{\text { â }}$ & 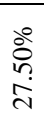 & $\frac{\delta_{0}^{\circ}}{\stackrel{0}{0}}$ & $\stackrel{\circ}{\curvearrowleft}$ & $\stackrel{\circ}{N}$ & $\begin{array}{l}\stackrel{0}{0} \\
\text { \&े } \\
\dot{8}\end{array}$ & 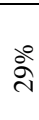 & 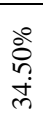 & 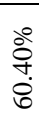 \\
\hline $\begin{array}{l}\text { Enterococcus } \\
\text { spp. }\end{array}$ & $=$ & 8 & $\stackrel{8}{8}$ & $\dot{8}_{0}^{\circ}$ & $\begin{array}{l}\stackrel{0}{0} \\
\stackrel{\circ}{n}\end{array}$ & 80 & $\stackrel{8}{8}$ & $\dot{\delta}_{n}^{\circ}$ & $\begin{array}{l}0 \\
\text { ô }\end{array}$ & $\dot{8}_{0}^{\circ}$ & ㅇํㅁ & $\delta_{0}^{\circ}$ \\
\hline $\begin{array}{l}\text { Citrobacter } \\
\text { spp. }\end{array}$ & $n$ & $\stackrel{8}{8}$ & $\stackrel{8}{\circ}$ & $\stackrel{8}{\circ}$ & $\stackrel{\circ}{\circ}$ & bீ & $\stackrel{8}{8}$ & $8_{0}^{\circ}$ & ठ̊ํ & $8_{0}^{\circ}$ & தீ & $\stackrel{0}{0}_{0}^{\circ}$ \\
\hline
\end{tabular}

\begin{tabular}{|c|c|c|c|c|c|c|c|c|c|c|c|c|c|c|c|c|c|c|}
\hline Name of organisms & $\begin{array}{l}\frac{0}{0} \\
\frac{\pi}{0} \\
0 \\
0 \\
0 \\
0 \\
\dot{z}\end{array}$ & 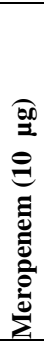 & 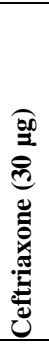 & 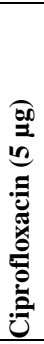 & 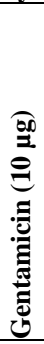 & 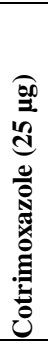 & 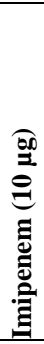 & 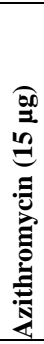 & 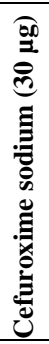 & 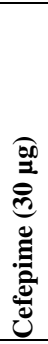 & 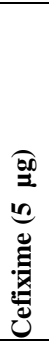 & 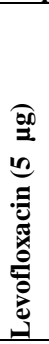 & 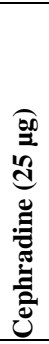 & 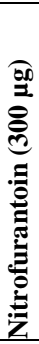 & 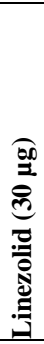 & 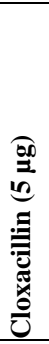 & 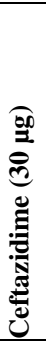 & 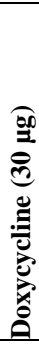 \\
\hline E. coli & 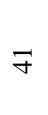 & $\stackrel{8}{8}$ & 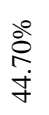 & $\begin{array}{l}\stackrel{0}{0} \\
\text { లి } \\
\text { in }\end{array}$ & $\begin{array}{l}0 \\
i n\end{array}$ & $\begin{array}{l}\stackrel{8}{\circ} \\
\stackrel{i}{\circ}\end{array}$ & $\stackrel{8}{8}$ & 之 & 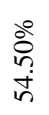 & $\begin{array}{l}\stackrel{\circ}{2} \\
\end{array}$ & $\begin{array}{l}\stackrel{8}{0} \\
\stackrel{0}{0} \\
\dot{n} \\
\text { n. }\end{array}$ & $\frac{\stackrel{8}{0}}{\stackrel{0}{0}}$ & 之 & $\frac{\stackrel{8}{0}}{\stackrel{0}{0}}$ & 之 & 之 & $\begin{array}{l}\stackrel{0}{\infty} \\
\infty\end{array}$ & \&̊ㅇ \\
\hline Staphylococcus aureus & ஓ & $\stackrel{8}{8}$ & $\begin{array}{l}\stackrel{0}{\Im} \\
\stackrel{y}{7}\end{array}$ & $\stackrel{\stackrel{0}{\circ}}{\stackrel{n}{n}}$ & $\begin{array}{l}\stackrel{0}{0} \\
\infty \\
\dot{0}\end{array}$ & 文 & $\begin{array}{l}\stackrel{0}{2} \\
\text { å }\end{array}$ & $\begin{array}{l}\stackrel{0}{ } \\
\infty \\
\dot{\infty} \\
\text { m. }\end{array}$ & $\begin{array}{l}\stackrel{0}{2} \\
\stackrel{\rho}{\circ} \\
\text { ले }\end{array}$ & $\stackrel{8}{8}$ & $\frac{\Delta}{m}$ & $\stackrel{\bigcirc}{\infty}$ & $\begin{array}{l}\stackrel{\circ}{n} \\
\text { m }\end{array}$ & $\begin{array}{l}\stackrel{0}{0} \\
\text { ஜn } \\
\text { ชூ }\end{array}$ & $\begin{array}{l}\stackrel{\circ}{\circ} \\
\stackrel{\circ}{\ominus}\end{array}$ & 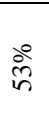 & $\begin{array}{l}\stackrel{0}{\circ} \\
\text { की }\end{array}$ & $\begin{array}{l}\stackrel{0}{0} \\
\infty \\
\text { ஸे } \\
\text { m. }\end{array}$ \\
\hline $\begin{array}{l}\text { Staphylococcus } \\
\text { epidermidis }\end{array}$ & $n$ & $\stackrel{8}{8}$ & $\frac{D^{\circ}}{d}$ & $\begin{array}{l}\stackrel{Ð}{0} \\
\infty \\
\text { ஸे }\end{array}$ & $\frac{\stackrel{\infty}{+}}{\stackrel{\infty}{+}}$ & 之 & $\stackrel{8}{8}$ & 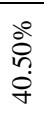 & $\begin{array}{l}\stackrel{0}{0} \\
\infty \\
0 \\
\stackrel{d}{0}\end{array}$ & $\frac{\stackrel{0}{\infty}}{\infty}$ & $\begin{array}{l}\stackrel{0}{0} \\
\stackrel{n}{2} \\
\text { }\end{array}$ & $\begin{array}{l}\stackrel{Ð}{0} \\
\stackrel{0}{+} \\
\text { ஸे }\end{array}$ & $\begin{array}{l}\stackrel{0}{0} \\
\stackrel{\circ}{n}\end{array}$ & 之 & 之 & $\begin{array}{l}\stackrel{0}{2} \\
\qquad 6\end{array}$ & $\begin{array}{l}\stackrel{0}{a} \\
\end{array}$ & $\stackrel{8}{+}$ \\
\hline Klebsiella pneumoniae & $\forall$ & $\stackrel{8}{8}$ & $\begin{array}{l}\stackrel{0}{n} \\
\stackrel{2}{n}\end{array}$ & $\frac{\stackrel{\infty}{\infty}}{\infty}$ & $\begin{array}{l}\delta_{0} \\
\text { ñ } \\
\text { in }\end{array}$ & 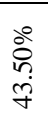 & $\frac{2}{5}$ & 之 & $\begin{array}{l}\stackrel{0}{0} \\
\stackrel{n}{?} \\
\stackrel{+}{+}\end{array}$ & $\frac{\circ}{2}$ & $\frac{\stackrel{0}{\ominus}}{\stackrel{i}{n}}$ & $\frac{\dot{D}^{\circ}}{\stackrel{8}{1}}$ & 之 & 之 & 之 & 之 & $\begin{array}{l}{ }_{0}^{0} \\
\stackrel{\circ}{n}\end{array}$ & $\begin{array}{l}\stackrel{0}{0} \\
\text { ஜn } \\
\stackrel{0}{0}\end{array}$ \\
\hline Salmonella spp. & $\forall$ & $\stackrel{8}{8}$ & $\begin{array}{l}\stackrel{0}{2} \\
i\end{array}$ & 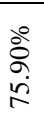 & 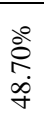 & $\begin{array}{l}\stackrel{0}{0} \\
\stackrel{\infty}{2} \\
\stackrel{2}{r}\end{array}$ & $\stackrel{8}{8}$ & 之 & $\begin{array}{l}\stackrel{2}{2} \\
\stackrel{2}{\circ}\end{array}$ & $\stackrel{8}{8}$ & $\stackrel{8}{\stackrel{f}{+}}$ & 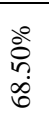 & 之 & 之 & $\begin{array}{l}\stackrel{0}{0} \\
\stackrel{0}{?} \\
\stackrel{+}{+}\end{array}$ & 之 & ठ̊ & 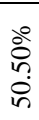 \\
\hline Streptococcus viridans & $N$ & $\stackrel{8}{8}$ & $\stackrel{\circ}{\circ}$ & $\begin{array}{l}\stackrel{\circ}{ } \\
\stackrel{0}{ } \\
\stackrel{+}{+}\end{array}$ & $\begin{array}{l}\stackrel{8}{0} \\
\text { } \\
\text { ஸூ }\end{array}$ & 之 & 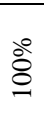 & $\begin{array}{l}\text { in } \\
\text { m }\end{array}$ & 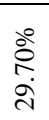 & $\stackrel{8}{8}$ & $\begin{array}{l}\stackrel{0}{0} \\
\stackrel{n}{0} \\
\stackrel{0}{0}\end{array}$ & $\frac{2}{6}$ & $\stackrel{\circ}{\circ}$ & 之 & $\begin{array}{l}\stackrel{\circ}{n} \\
\stackrel{1}{n}\end{array}$ & ठ̊ & $\stackrel{\stackrel{0}{0}}{\stackrel{0}{2}}$ & $\begin{array}{l}\delta^{\circ} \\
0 \\
n \\
0 \\
m\end{array}$ \\
\hline
\end{tabular}


Table 3: Antibiotic Sensitivity of bacterial isolates collected from stool specimens.

\begin{tabular}{|c|c|c|c|c|c|c|c|c|c|c|c|c|}
\hline $\begin{array}{l}\text { Name of } \\
\text { organisms }\end{array}$ & 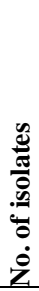 & 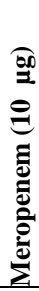 & 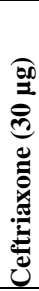 & 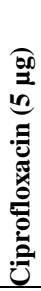 & 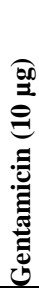 & 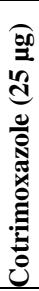 & 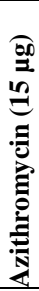 & 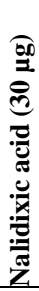 & 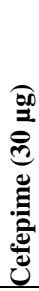 & 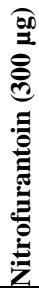 & 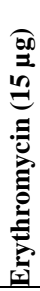 & 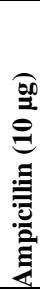 \\
\hline E. coli & $\stackrel{\sim}{\sim}$ & $\stackrel{8}{8}$ & 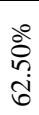 & $\begin{array}{l}\stackrel{d}{0} \\
\stackrel{\infty}{\infty} \\
i n\end{array}$ & $\stackrel{8}{n}$ & $\frac{\stackrel{0}{0}}{\stackrel{0}{\sigma}}$ & $\begin{array}{l}\delta_{0}^{0} \\
\mathscr{D}_{0} \\
\stackrel{0}{0}\end{array}$ & $\stackrel{8}{2}$ & $\frac{\stackrel{0}{0}}{\stackrel{\infty}{\infty}}$ & $\frac{\stackrel{\partial}{0}}{\stackrel{\infty}{\infty}}$ & $\begin{array}{l}8 \\
0 \\
0 \\
0 \\
0\end{array}$ & $\begin{array}{l}0_{0}^{0} \\
\infty \\
\infty\end{array}$ \\
\hline $\begin{array}{l}\text { Shigella } \\
\text { spp. }\end{array}$ & $a$ & ᄋ̊ & $\begin{array}{l}\stackrel{0}{\circ} \\
\infty \\
\infty \\
\infty\end{array}$ & $\begin{array}{l}\delta_{0}^{0} \\
\overbrace{0} \\
\stackrel{0}{0}\end{array}$ & 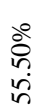 & 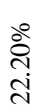 & $\frac{\stackrel{2}{2}}{\stackrel{2}{R}}$ & 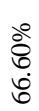 & $\begin{array}{l}\stackrel{0}{0} \\
\infty \\
\infty \\
\infty\end{array}$ & $\begin{array}{l}\stackrel{0}{0} \\
\infty \\
\infty \\
\infty\end{array}$ & $\begin{array}{l}\stackrel{0}{8} \\
\stackrel{+}{+} \\
\dot{y}\end{array}$ & $\frac{\stackrel{d}{0}}{\stackrel{0}{m}}$ \\
\hline $\begin{array}{l}\text { Vibrio } \\
\text { cholerae }\end{array}$ & $r$ & 8̊ & $\begin{array}{l}\frac{2}{2} \\
\stackrel{2}{\infty} \\
\dot{\infty}\end{array}$ & $\frac{\stackrel{d}{\circ}}{\stackrel{f}{r}}$ & $\begin{array}{l}\stackrel{0}{0} \\
\infty \\
\text { ㅁ } \\
\text { f }\end{array}$ & $\begin{array}{l}\stackrel{0}{尺} \\
\stackrel{\infty}{\infty}\end{array}$ & $\frac{\stackrel{b}{0}}{i n}$ & $\frac{\stackrel{8}{0}}{\stackrel{8}{+}}$ & $\stackrel{8}{8}$ & $\begin{array}{l}\stackrel{2}{尺} \\
\stackrel{2}{\infty}\end{array}$ & $\begin{array}{l}\stackrel{0}{0} \\
\stackrel{0}{ } \\
\text { ㅁ }\end{array}$ & 80 \\
\hline $\begin{array}{l}\text { Salmonella } \\
\text { spp. }\end{array}$ & $\nabla$ & 8 & $\dot{D}_{n}^{0}$ & $\frac{D^{\circ}}{n}$ & $\stackrel{8}{n}$ & $\dot{0}^{0}$ & $\delta_{0}^{\circ}$ & $\grave{0}_{n}^{0}$ & $\stackrel{8}{8}$ & $\frac{8}{n}$ & $\begin{array}{l}\infty^{2} \\
\text { in }\end{array}$ & 80 \\
\hline
\end{tabular}

Table 4: Antibiotic sensitivity of bacterial isolates collected from pus specimens.

\begin{tabular}{|c|c|c|c|c|c|c|c|c|c|c|c|c|c|c|c|c|}
\hline Name of organisms & $\begin{array}{l}\frac{0}{\tilde{n}} \\
\frac{0}{0} \\
\frac{0}{0} \\
\dot{0} \\
\dot{0}\end{array}$ & 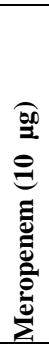 & 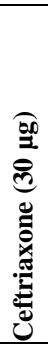 & 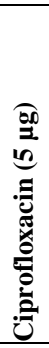 & 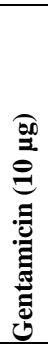 & 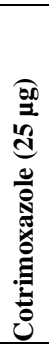 & 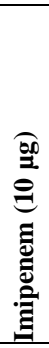 & 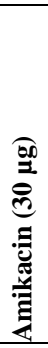 & 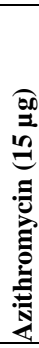 & 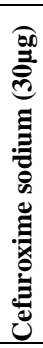 & 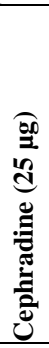 & 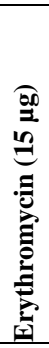 & 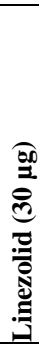 & 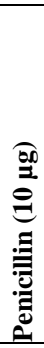 & 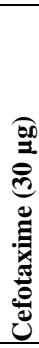 & 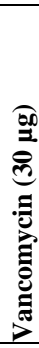 \\
\hline Staphylococcus aureus & ฉે & চ̊ㅇ & $\stackrel{8}{8}$ & $\begin{array}{l}\stackrel{\circ}{\sim} \\
\stackrel{\sim}{n}\end{array}$ & $\stackrel{8}{\circ}$ & চి & $\stackrel{8}{8}$ & $\stackrel{8}{0}$ & \&̊ำ & 官 & $\stackrel{8}{\circ}$ & $\stackrel{\infty}{\infty}$ & ஓ̊ & 8 & b̊ & ठ্ণ \\
\hline E. coli & $\infty$ & $\begin{array}{l}8 \\
8\end{array}$ & $\stackrel{\circ}{\circ}$ & 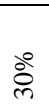 & 仓̊ & $\stackrel{8}{\circ}$ & ㅇํㅇ & 80 & $\stackrel{8}{\circ}$ & ஃீ & $\stackrel{\circ}{\circ}$ & $\stackrel{8}{0}$ & 追 & \&̊ & : & $\stackrel{\infty}{\infty}$ \\
\hline Pseudomonas spp. & $\infty$ & $\stackrel{8}{\circ}$ & $\stackrel{\circ}{\infty}$ & চ্ & $\stackrel{8}{8}$ & 용 & $\stackrel{8}{8}$ & 80 & $\begin{array}{l}80 \\
\stackrel{\circ}{\circ}\end{array}$ & $\stackrel{\infty}{\infty}$ & $\grave{\infty}_{\infty}^{0}$ & $\stackrel{8}{0}$ & $\begin{array}{l}80 \\
8 \\
0\end{array}$ & 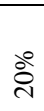 & $\stackrel{\circ}{\circ}$ & : \\
\hline Acinetobacter spp. & $\nabla$ & $\frac{8}{8}$ & $\stackrel{8}{8}$ & $\stackrel{8}{8}$ & $\stackrel{80}{0}$ & $\stackrel{\circ}{\circ}$ & $\stackrel{8}{8}$ & $\stackrel{8}{8}$ & $\stackrel{8}{0}_{0}^{\circ}$ & $\stackrel{8}{8}$ & $\begin{array}{l}\stackrel{0}{0} \\
\stackrel{\circ}{n}\end{array}$ & 仓̊ & $\stackrel{\infty}{\infty}_{0}^{\circ}$ & $\mathrm{z}$ & $\stackrel{8}{0}$ & $\stackrel{\circ}{\circ}$ \\
\hline Klebsiella spp. & 一 & $\stackrel{\circ}{\infty}$ & ஃ̊ & ঠ̊ㅇ f & $\begin{array}{l}\stackrel{0}{\circ} \\
\stackrel{n}{n}\end{array}$ & ஃ̊ & $\stackrel{8}{8}$ & 之 & 之 & 之 & $\begin{array}{l}80 \\
\text { ஜ্ }\end{array}$ & ठ̊ & $\stackrel{8}{\circ}$ & $\frac{\stackrel{0}{\circ}}{\grave{n}}$ & 之 & $\stackrel{8}{8}$ \\
\hline
\end{tabular}

\section{DISCUSSION}

According to our data, females were accounted for $57.10 \%(n=197)$ of all the patients with UTIs. In fact, women get UTIs up to 30 times more than men. Around 50$60 \%$ of women develop at least one UTI episode in their lifetime due to a combination of factors like the anatomical differences as the shortened urethra and more sensitive skin than male, reproductive life cycles, menopause, pregnancy, etc. (912). Conversely, males were more prone to other infections that were visible with the positive cultures from blood, stool, and pus specimens that are correlated with previously published studies $(3,13,14)$. In any of the specimens, most positive cases were from adult (31-40 years) patients 
except in the case of stool samples where most of the positive cases were from the early ages $(0-10$ years $)(10,11)$. This can be described as around 3-5 billion cases of acute gastroenteritis, and 2 million deaths occur under 5 years old children every year worldwide (15). Children are easily getting infected due to contaminated water and food as well as by contacting contaminated surfaces or animals that may carry certain bacteria like Salmonella spp. (16-18). Most positive cases were reported in summer-rainy seasons except in pus where the highest count was observed in late autumn. In summer, the atmosphere is suddenly changed as the heat and humidity rise which might be responsible to create unhygienic conditions, a source of contamination of water and food which is easier for pathogenic growth. It is also easier to lose fluid and those who do not stay hydrated could have a higher risk of getting UTIs. Moreover, geographical location, climate condition, and lack of hygienic practice are responsible for the causes of diseases (19-21).

In our study, E. coli bacteria was accounted very frequently $(53.04 \%$ in urine, $36.28 \%$ in blood, and $54.55 \%$ in stool samples) which relates to other studies like $31.4 \%$ in Cameroonian towns (11), 34.1\% in Namibia (12) and $86 \%$ in Nepal (10) were detected in the specimen of urine. Likewise, E. coli is the most common etiology of diarrhea among children in Bangladesh as well as in other developing countries resembling India $(44.2 \%)$ and Sudan (53.8\%) (17). Even in a blood specimen, the count of E. coli was the highest $(\mathrm{n}=41,36.28 \%)$ where Simkhada et al. in 2016 described Salmonella Paratyphi A $(n=26,54.17 \%)$ as the leading etiological agent in blood-borne infections and $E$. coli is only $6.25 \% \quad(n=3) \quad(22)$. The most common isolate in pus specimen was Staphylococcus aureus $(\mathrm{n}=29, \quad 58 \%)$ followed by $E$. coli $(\mathrm{n}=8,16 \%)$ which correlates with previous studies $(18,23)$. Although in some instances, E. coli was the most frequently detected bacteria in pus specimens $(6,21)$. As a normal flora, E. coli is commonly found in the gut of warmblooded organisms but due to some circumstances, it becomes pathogenic.

Most of the gram-positive and gramnegative bacteria showed a high degree of sensitivity towards Meropenem. Even, some of those showed a good degree of sensitivity towards Imipenem and Cefepime. Both Meropenem and Imipenem are from the carbapenem class of antibiotics and their overall spectrum is very similar as both act against gram-positive and gram-negative bacteria although Meropenem is more active against Enterobacteriaceae than gramnegative bacteria (24). Furthermore, Cefepime is a $4^{\text {th }}$ generation cephalosporin antibiotic that has an extended spectrum of activity against both gram-positive and gram-negative bacteria $(25,26)$. The sensitivity of both gram-positive and gramnegative bacteria towards those antibiotics was reported previously in several research works $(3,9)$. The most alarming scenario is the generation of highly resistant bacteria species against 3rd generation (like Cefotaxime, Ceftazidime, Ceftriaxone, Cefixime) and even, $4^{\text {th }}$ generation (Cefepime) Cephalosporin antibiotics. The increasing generation of drug-resistant bacteria against $3^{\text {rd }}$ and $4^{\text {th }}$ generation Cephalosporin antibiotics was reported previously that could render therapy against bacterial infections unsuccessful (27-30).

If more stool and pus samples could be reported that would be good for our study. However, due to the unavailability of all of the antibiotics throughout the study period, susceptibility tests were not uniformly performed by all the isolates which made it quite difficult to compare the pattern of sensitivity or resistance by all the isolates. However, an alarming scenario was that more than $90 \%$ of the isolates were resistant to more than 3 antibiotics.

\section{CONCLUSION}

Although drug resistance of common infectious bacteria is very distressing in the treatment of infectious diseases, it seems 
like the issue will not tint until it would be very late. More specifically, in a developing country like Bangladesh, it is required to take immediate action, and implement laws and regulations to combat the most imminent biological threat to human civilization. Our study emphasizes the emergence of drug resistance against even the latest generations of antibiotics as they are used uncontrollably and unconsciously that poses scope for the generation of drug resistance in bacteria. Along with the systematic regional study to view the current scenario of drug resistance and governmental and nongovernmental actions, it is also required to search for natural or to generate synthetic or semi-synthetic antibiotics.

Conflict of Interest: There is no conflict of interest.

\section{Acknowledgement: None}

\section{Source of Funding: None}

\section{Ethical Approval: Approved}

\section{REFERENCES}

1. Nii-Trebi NI. Emerging and Neglected Infectious Diseases: Insights, Advances, and Challenges. BioMed Res Int. 2017;2017:5245021.

2. Khatun MS, Nahar S, Kabir MS. Antibiotic resistance pattern of bacteria isolated from outdoor patients in Dhaka city: a singlecenter study. Stamford J Microbiol. 2019;9(1):1-4.

3. Mia AR, Zerin, T. Antibiogram of blood culture isolates of patients from a hospital in Dhaka, Bangladesh. Matrix Science Medica. 2020;4(1):1-5.

4. Vazquez-Pertejo MT. Diagnosis of Infectious Disease. MSD MANUL Consumer Version. Last full review/revision in Jun 2020. Content last modified in Jun $2020 . \quad$ Available at, https://www.msdmanuals.com/home/infecti ons/diagnosis-of-infectious disease/diagnosis-of-infectious-disease.
5. WHO. Antibiotic resistance. 31 July, 2020. Available at, https://www.who.int/newsroom/fact-sheets/detail/antibiotic-resistance.

6. Zerin T, Islam A, Gulnahar S, Farjana NE, Begum MA, Sadia H-E. Identification and Antibiotic Susceptibility of Blood Culture Isolates from Rajshahi, Bangladesh. JSRMBS. 2021;2(2):1-10.

7. Collee JG, Miles RS, Watt B. Tests for the Identification of Bacteria. In: Collee, J.G., Marmion, B.P., Fraser, A.G., \& Simmons, A., Eds., Mackie \& McCartney Practical Medical Microbiology, 14th Edition, Churchill Livingstone, New York; 1996.p.131-151

8. 8. CLSI. Performance Standards for Antimicrobial Susceptibility Testing. Twenty-Sixth Informational Supplement. CLSI document M100-S26. Wayne (PA);2016:50-6.

9. Farjana NE, Islam MA, Zerin T, Begum MA. Bacterial association in urinary tract infection and their drug resistance among patients in Rajshahi, Bangladesh. IJCMPH. 2021; 8:2144.

10. Subedi N, Pudasaini S. (2017). Bacteriological profile and antibiotic sensitivity pattern in patients with Urinary tract infection. J Pathol Nepal. 2017;7(1): 1066-1069.

11. Akoachere JF, Yvonne S, Akum NH, Seraphine EN. (2012). Etiologic profile and antimicrobial susceptibility of communityacquired urinary tract infection in two Cameroonian towns. BMC res notes. 2012;5:219.

12. Mengistu AT, Forster N, Gaeseb J, Habimana G, Kauhondamwa S, Ndjavera C, et al. A Review of Empirical Treatment of Urinary Tract Infections Based on National Antimicrobial Sensitivity Data. Enliven: Pharmacovigil Drug Saf. 2014;1(1):002.

13. Qu M, Lv B, Zhang X, Yan H, Huang Y, Qian $\mathrm{H}$, et al. Prevalence and antibiotic resistance of bacterial pathogens isolated from childhood diarrhea in Beijing, China (2010-2014). Gut pathog. 2016;8:31.

14. Shewly SR, Ane MHA, Zerin T, Hossain MA. Antibiotic Susceptibility of Bacterial Isolates from Pus Specimens Collected from a General Hospital in Dhaka, Bangladesh. Int J Res Sci Innov. 2021; VIII(VII):62-66.

15. Elliott EJ. Acute gastroenteritis in children. Br Med J (Clin res ed.). 2007;334(7583):3540. 
16. Cochran WJ. Gastroenteritis in Children. MSD MANUAL, Consumer Version. Last full review/revision Aug 2021. Available at, https://www.msdmanuals.com/home/childre n-s-health-issues/digestive-disorders-inchildren/gastroenteritis-in-children.

17. Pervin MK, Jhora ST, Paul S, Naher A. Antibiotic sensitivity pattern of diarrhoeal pathogens in under-five children. Bangladesh Med J Khulna. 2020;52:35-39.

18. Singh A, Verma V, Singh R. Antibiotic Sensitivity Pattern of Pathogens Isolated from Pus Culture- A Tertiary Care Hospital Based Study. J Nepalgunj Med Coll. 2019;17(2):70-74.

19. Fares A. Factors influencing the seasonal patterns of infectious diseases. Int $\mathbf{J}$ prev med. 2013;4(2):128-132.

20. Demissie TA, Wubie MT, Yehuala FM, Fetene DM, Gudeta GA. (2014). Prevalence and antimicrobial susceptibility patterns of Shigella and Salmonella Species among patients with diarrhea attending Gondar town health institutions, Northwest Ethiopia. SJPH. 2014;2(5):469-475.

21. Trojan R, Razdan L, Singh N. (2016). Antibiotic Susceptibility Patterns of Bacterial Isolates from Pus Samples in a Tertiary Care Hospital of Punjab, India. Int J Microbiol. 2016;2016:9302692.

22. Simkhada P, Raj KCS, Lamichhane S, Subedi S, Shrestha UT. Bacteriological profile and antibiotic susceptibility pattern of a blood culture isolate from patients visiting Tertiary Care Hospital in Kathmandu, Nepal. Glob J Med Res. 2016; 16(1):25-31.

23. Pandeya U, Raut M, Bhattarai S, Bhatt PR, Dahal PR. Bacteriological Profile and Antibiogram of Bacterial Isolates from Pus Samples in Tertiary Care Hospital of Kathmandu. TUJM. 2018;4:55-62.
24. McEvoy GK. AHFS Drug Information. Bethesda: The American Society of HealthSystem Pharmacists, Inc., 2006.

25. Yahav D, Paul M, Fraser A, Sarid N, Leibovici L. Efficacy and safety of cefepime: a systematic review and metaanalysis. The Lancet. Infect dis. 2007;7(5): 338-348.

26. Wynd MA, Paladino JA. Cefepime: a fourth-generation parenteral cephalosporin. Ann Pharmacother. 1996;30(12):1414-1424.

27. Gashe F, Mulisa E, Mekonnen M, Zeleke G. Antimicrobial Resistance Profile of Different Clinical Isolates against ThirdGeneration Cephalosporins. J pharm. 2018; 2018:5070742.

28. Moghnieh R, Estaitieh N, Mugharbil A, Jisr T, Abdallah DI, Ziade F. Third-generation cephalosporin-resistant Enterobacteriaceae and multidrug-resistant gram-negative bacteria causing bacteremia in febrile neutropenia adult cancer patients in Lebanon, broad-spectrum antibiotics use as a major risk factor, and correlation with poor prognosis. Front. Cell. Infect. Microbiol. 2015;5:11.

29. Fair RJ, Tor Y. Antibiotics and bacterial resistance in the 21st century. Perspect Medicin Chem. 2014;6:25-64.

30. Breurec S, Bouchiat C, Sire JM, Moquet O, Bercion R, Cisse MF, et al. High thirdgeneration cephalosporin-resistant Enterobacteriaceae prevalence rate among neonatal infections in Dakar, Senegal. BMC Infect Dis. 2016;16:587.

How to cite this article: Shekha Nita Mondal, Md. Ariful Islam, Tamanna Zerin. Characterization and antibiotic susceptibility of bacteria isolated from clinical specimens in Rajshahi, Bangladesh. Int J Health Sci Res. 2022; 12(2):16-25. DOI: https://doi.org/10. 52403/ijhsr.20220203 